\title{
e-Phaïstos
}

e-Phaïstos

Revue d'histoire des techniques / Journal of the history

of technology

I-2 | 2012

Les sources de l'histoire des techniques

\section{Sources et méthodes pour une histoire des techniques métallurgiques anciennes dans les sociétés africaines subsahariennes : le cas de la métallurgie du fer}

Hélène Timpoko Kiénon Kabore

\section{OpenEdition}

Édition électronique

URL : http://journals.openedition.org/ephaistos/403

DOI : 10.4000/ephaistos.403

ISSN : 2552-0741

Éditeur

IHMC - Institut d'histoire moderne et contemporaine (UMR 8066)

Édition imprimée

Date de publication : 1 décembre 2012

Pagination : $28-40$

ISSN : 2262-7340

\section{Référence électronique}

Hélène Timpoko Kiénon Kabore, «Sources et méthodes pour une histoire des techniques

métallurgiques anciennes dans les sociétés africaines subsahariennes : le cas de la métallurgie du fer », e-Phaïstos [En ligne], I-2 | 2012, mis en ligne le 12 février 2016, consulté le 01 mai 2019. URL : http:// journals.openedition.org/ephaistos/403; DOI : 10.4000/ephaistos.403 


\title{
Sources et méthodes pour une histoire des techniques métallurgiques anciennes dans les sociétés africaines subsahariennes : le cas de la métallurgie du fer
}

\author{
Hélène-Timpoko Kiénon-Kabore \\ Département d'Archéologie: \\ Institut des Sciences Anthropologiques de Dé- \\ veloppement (ISAD) \\ Université Félix Houphouët-Boigny de Cocody \\ Abidjan, Côte d'Ivoire
}

\section{Introduction}

La métallurgie ancienne du fer en Afrique subsaharienne a été le centre d'intérêt de plusieurs disciplines. Sociologues, anthropologues, ethnologues, historiens, archéologues se partagent les investigations sur cette thématique. Ces recherches entraînent de véritables débats scientifiques.

Les travaux de certains auteurs ${ }^{1}$ du début au milieu du XX ${ }^{\mathrm{e}}$ siècle ont nié à l'Afrique l'invention de la métallurgie du fer. Ils ont présenté l'Afrique comme un continent qui a emprunté ses techniques sidérurgiques aux autres civilisations par des voies diffusionnistes. Les recherches des spécialistes qui ont suivi ont montré que l'Afrique en général et particulièrement l'Afrique subsaharienne, a connu la métallurgie ancienne du fer et possède également une industrie autochtone très riche qui a contribué à l'édification du patrimoine technique mondial ${ }^{2}$.

Ces résultats ont été possibles grâce à des sources diversifiées et des méthodes rigoureuses d'approche qui ont permis de répondre aux différentes préoccupations scientifiques. Les études sur la métallurgie du fer en Afrique subsaharienne ont leurs particularités qui né- cessitent le plus souvent une approche spécifique. Quels sont les apports des sources matérielles ? Comment aborder l'histoire de la métallurgie ancienne du fer dans des sociétés où les traditions orales sont des sources de bases ? Que faire pour tirer des informations des sources immatérielles liées à la métallurgie qui renferment une grande partie des informations historiques? Comment pénétrer et comprendre ce langage d'initié quand on est profane?

Ces questions nécessitent une triple approche : les sources matérielles, les sources orales et les sources immatérielles.

\section{Les sources matérielles}

Les sources matérielles se composent de documents écrits, de vestiges archéologiques et de vestiges historiques. Ces éléments ont été très déterminants dans l'approche et la compréhension des techniques métallurgiques du fer en Afrique subsaharienne.

\section{Les documents écrits}

Les recherches sur l'histoire des techniques métallurgiques en Afrique subsaharienne sont difficiles à 
cause de la rareté des documents écrits, bien qu'à ce jour, des pays africains comme le Burkina-Faso, le Sénégal, le Nigéria, etc. ont pu en trois décennies réunir une documentation écrite importante ${ }^{3}$. Plusieurs pays africains sont restés en marge de ces recherches et n'ont aucune documentation scientifique exploitable sur le sujet. On ne dispose, quelquefois, que de rares articles écrits de façon isolée, en dehors de véritables programmes de recherche. Par conséquent, il est nécessaire de recourir aux documents anciens (sources d'archives). $\mathrm{Au}$ sein de cette documentation, on trouve le plus souvent des écrits d'explorateurs, qui constituent de véritables mines d'informations. On peut citer les écrits du
Capitaine Binger ${ }^{4}$ à la fin du XIX ${ }^{\mathrm{e}}$ siècle, d'une grande richesse descriptive pour les industries et les techniques de certaines populations et souvent accompagnés de dessins très instructifs. Ainsi, il présente un croquis accompagné de descriptions qui montre une scène de réduction directe du minerai de fer par batterie au Mali et au Burkina-Faso (fig. 1). L'on perçoit nettement plusieurs types de fourneaux en activité, dont des fourneaux cylindriques, tronconiques et cubiques. L'image et les descriptions qui l'accompagnent donnent des informations sur les modes de chargement, les métallurgistes, les typologies de fourneaux, les types d'ouvertures à la base, l'alimentation en air, l'évacuation

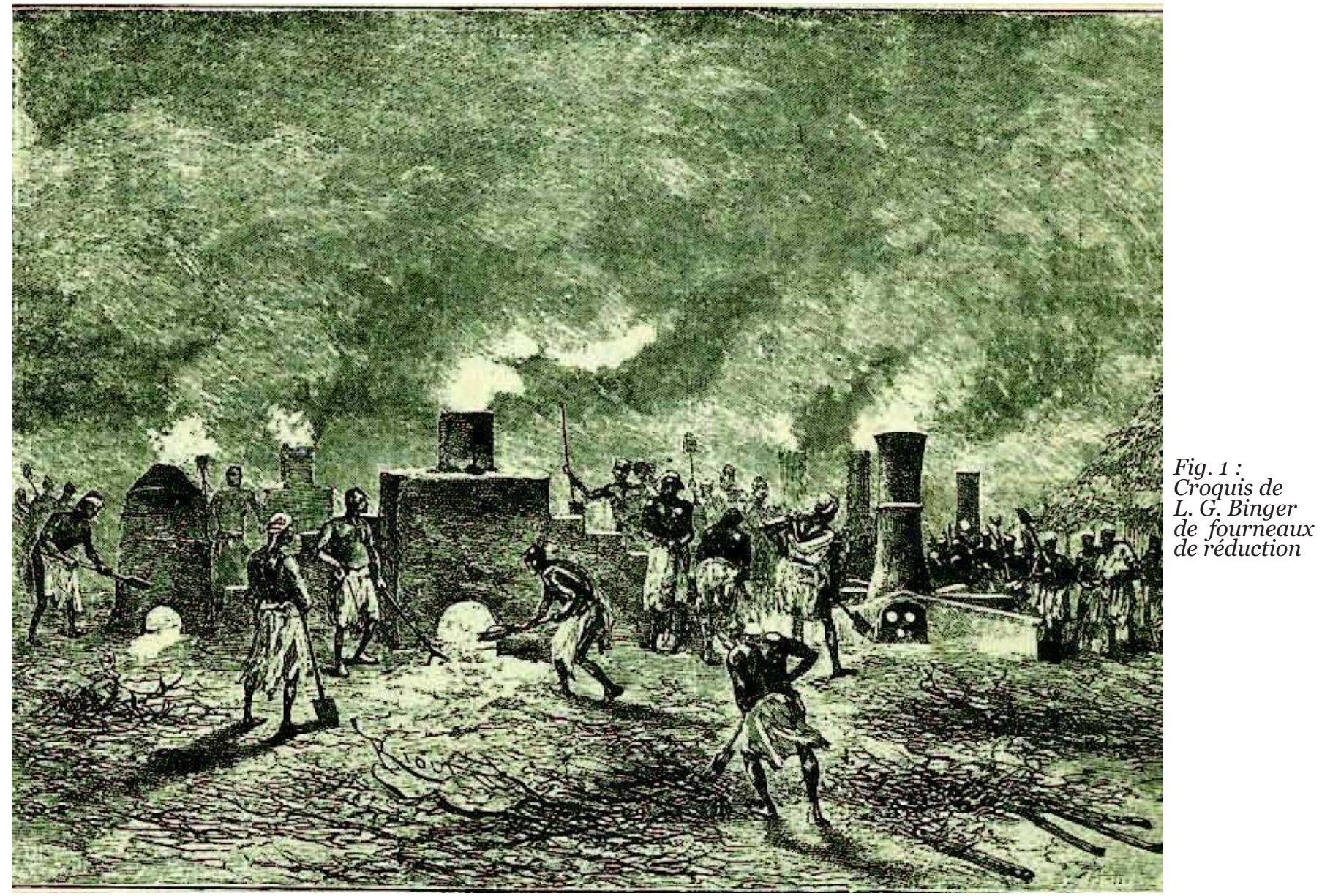


des scories, les types d'ouverture au sommet destinées au chargement et à l'évacuation de la chaleur.

J. Meniaud ${ }^{5}$ présente également dans son livre une photographie qui met en évidence une aire de réduction directe du minerai de fer dans le Yatenga au nord du Burkina-Faso actuel (fig. 2). On y voit également plusieurs fourneaux de forme cylindrique, d'environ quatre mètres de haut chacun. À la base, on perçoit plusieurs ouvertures servant à la ventilation et à l'évacuation des scories.

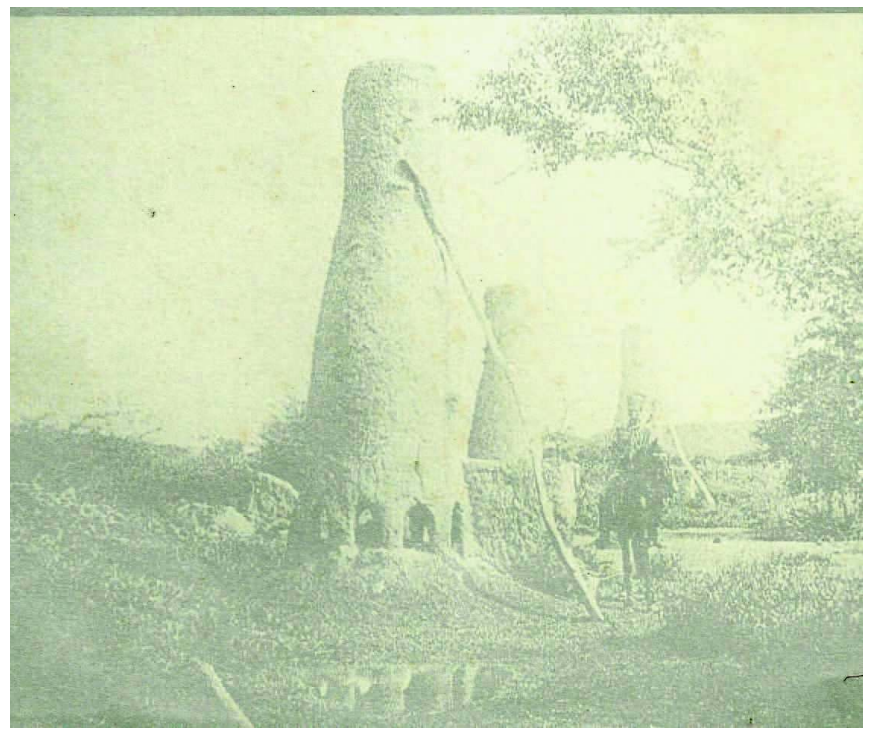

Fig. 2 : Photographie de J. Meniaud de fourneaux de réduction

Félix Yandia signale aussi l'importance des sources écrites anciennes dans les recherches sur la métallurgie traditionnelle du fer en République Centrafricaine ${ }^{6}$. Il présente les écrits des explorateurs C. Maître et Dybowsky. Le premier donne en 1893 des informations sur la réputation en matière de métallurgie du fer des populations Gombé et Ndriss. Il décrit aussi des fourneaux et forges. Le second signale des fourneaux dans la forêt proche de Makorou. Ces informations ont été essentielles pour F. Yandia.
Au Bénin, les mêmes sources sont indiquées pour la connaissance des techniques métallurgiques anciennes ${ }^{7}$. Les explorateurs Skertchly et Foa, après avoir visité le royaume Danxomé, ont révélé l'existence de fourneaux de réduction du mirerais de fer : Skertchly en septembre 1871 a vu fonctionner un four, et des années plus tard, Foa (de juin 1886 à mars 1890) donne aussi des descriptions de fourneaux.

Pendant la période coloniale, les monographies de cercles, les rapports des administrateurs coloniaux, des géologues, des amateurs, etc. donnent aussi des informations qui peuvent souvent s'avérer décisives dans la collecte des données préliminaires. Au sein de ceux-ci, des sites de métallurgistes sont souvent signalés sur des cartes et des photographies. Des auteurs y décrivent également des industries métallurgiques. Cette approche permet, le plus souvent, de mettre en place une première cartographie générale des sites archéologiques et d'avoir ainsi une évaluation de la richesse des sites métallurgiques de la zone d'étude afin de préparer les prospections de terrain.

En Côte d'Ivoire, des amateurs de la période coloniale ont fait des découvertes fortuites à l'exemple de Siméoni et Riester. En 1929, Riester, cité par Lemassou Fofana, signale l'existence d'un site métallurgique à Oumé qu'il assimile à une production carthaginoise vu l'importance des vestiges ${ }^{8}$. Il estime la quantité produite à plus de 10 ooo tonnes. Ces premiers écrits, même s'ils ne sont pas scientifiques, donnent des indications sur les localisations des sites et l'ampleur de la production métallurgique, souvent essentielles pour les études préalables à entreprendre sur le terrain.

Mis à part cette première catégorie de sources d'archives, il faut aussi prendre en compte les premiers écrits de certaines spécialités telles que l'ethnologie, l'anthropologie et les premières approches archéologiques.

En effet, à la mise en place des administrations coloniales au début du $\mathrm{XX}^{\mathrm{e}}$ siècle, ces disciplines connaissent un développement certain. Les administrations devant connaître les populations qu'elles maintenaient sous leurs tutelles pour leurs propres intérêts. Il fallait 
une meilleure connaissance des pays, des hommes, des ressources, des cultures et des environnements afin d'entamer « la mission civilisatrice ». La création du Comité d'étude historique et scientifique de l'AOF (Afrique-Occidentale française) destiné à coordonner les recherches entreprises a enregistré un grand dynamisme des recherches en archéologie. Ses actions constitueront le fer de lance des recherches archéologiques en Afrique-Occidentale française. Ce comité, même si l'idéologie était de servir l'intérêt de la colonisation, va s'illustrer par le sérieux de ses recherches et jeter ainsi les bases de la création de l'IFAN (l'Institut français d'Afrique noire) qui fut un remarquable instrument de recherche et l'agent le plus actif de l'émergence d'un pôle archéologique crédible en Afrique de l'Ouest ${ }^{9}$. Les acteurs scientifiques sont nombreux. On peut citer Maurice Délafosse, Clozel, le Docteur P. Jouenne, P. Laforgue, F. Zeltner, R. Furon, G. Watrelot, Th. Monod, R. Mauny etc. Les écrits ne concernent pas spécifiquement la métallurgie du fer, mais certains abordent les thèmes relatifs à l'âge du fer, ou donnent des indications indispensables à l'histoire des techniques métallurgiques en Afrique subsaharienne. Laforgue par exemple, qui a travaillé sur la préhistoire et l'âge des métaux, est une source indispensable pour l'étude de la métallurgie.

Ces documents qui concernent la fin du XIX ${ }^{\mathrm{e}}$ siècle et s'étendent à la deuxième moitié $\mathrm{du} \mathrm{XX}^{\mathrm{e}}$ siècle permettent aux spécialistes d'avoir des éléments indispensables pour les premières ébauches de travail sur le terrain. Ces derniers confrontent ces informations avec les données complémentaires recueillies auprès des populations au sujet des sites métallurgiques et des techniques utilisées, restées la plupart du temps muettes à cause de la rareté des documents écrits.

Ces sources anciennes ont permis de mettre en place des écrits scientifiques grâce aux spécialistes qui ont, au cours de ces trois dernières décennies, apporté beaucoup à la connaissance de l'histoire des techniques métallurgiques du fer en Afrique subsaharienne. Ces scientifiques sont des chercheurs africains, européens, américains, etc. Les documents sont des repères pour tous ceux qui veulent mener une étude scientifique sur la métallurgie ancienne du fer en Afrique subsaharienne, car ils présentent des méthodes, des approches et des résultats sur lesquels on peut se baser pour aborder de nouvelles études sur des terrains vierges ou à peine entamés comme ceux de l'Afrique subsaharienne.

Pour les périodes plus anciennes, les vestiges archéologiques sont les sources de base sur lesquelles se fonde l'historien des techniques. Ils lui permettent de faire une étude sur la longue durée afin de comprendre ce passé technique et appréhender les techniques plus récentes.

Les vestiges archéologiques, historiques et l'approche archéométrique

Les vestiges archéologiques et historiques sont des témoins matériels visibles de l'industrie sidérurgique. Ils témoignent de cette histoire technique allant des restes de la recherche des matières premières jusqu'à l'obtention des produits finis. Les vestiges historiques sont souvent les restes de production métallurgique des périodes récentes dont une grande partie se compose d'objets finis toujours utilisés par les forgerons et les populations actuelles.

Dans tous les pays de l'Afrique subsaharienne, les vestiges archéologiques de la métallurgie du fer ont été découverts. Aucun pays d'Afrique n'est resté en marge de cette révolution technique.

Les vestiges découverts ont fourni des datations très anciennes qui confirment l'ancienneté de cette industrie dans cette partie de l'Afrique.

Dans la région de Nsukka au Nigeria, la métallurgie a été datée de 760 avant notre ère à 1950 après notre ère. Plus de 2500 ans d'activité métallurgique. Ce qui fait de cette région l'un des centres les plus anciens de la métallurgie du fer en Afrique ${ }^{10}$. Des datations anciennes ont aussi été obtenues sur des sites archéologiques de la métallurgie du fer au Niger. Gérard Quéchon donne deux dates essentielles. Les objets en fer et en cuivre font leur apparition à Termit à peu près en 1500 avant notre ère, les premiers fourneaux de métallurgie sont datés aux environs de 800 avant notre ère ${ }^{11}$. 
Les vestiges archéologiques sont très nombreux en Afrique subsaharienne et concernent toute la chaîne opératoire de la sidérurgie directe. Cela permet, à partir des vestiges laissés en place, de reconstituer cette technique en prenant en compte toutes les étapes. Les documents écrits étant rares et quelquefois inexistants, les vestiges constituent des sources fiables qui peuvent combler le manque par des descriptions et des analyses scientifiques basées sur une méthodologie adéquate.

Les sites d'extraction du minerai de fer nous informent sur les modes d'extraction qui sont très divers. L'histoire des techniques minières est très riche en informations sur les types de minerai, les types d'exploitation, les types de mines, les différents systèmes de circulation, les modes de préparation du minerai, l'organisation du travail des mineurs, les modes d'acquisition et de vente du minerai, etc.

Au Burkina Faso, les vestiges de l'exploitation minière sont très nombreux et les types de mines très divers. Dans presque toutes les provinces, on rencontre des vestiges anciens de l'extraction du minerai de fer, allant du puits circulaire aux grandes fosses d'extraction.

L'extraction se faisait d'une part sur les surfaces cuirassées des plaines, des chaînes de collines, des sommets de montagnes et d'autre part sur les bords des fleuves ${ }^{12}$. La recherche du minerai se faisait aussi par ramassage simple de surface, ce qui ne laissait pas de traces archéologiques visibles. Par contre les autres formes ont le plus souvent laissé des traces.

Ce sont par exemple les exploitations par puits circulaires très utilisés sur certaines collines et plaines cuirassées. En les observant, on y voit des systèmes de circulation à spirales, à encoches et à cran ${ }^{13}$. Il y a également les extractions sur les collines et montagnes qui peuvent se faire soit à ciel ouvert, par décapage, soit par des excavations qui laissent des formes carrées ou rectanglulaires.

Une datation a été obtenue à Bena dans la province de la Kossi sur des puits de mine de fer entre -365 et $-22 \mathrm{O}^{14}$.

En Côte d'Ivoire, les traces archéologiques de l'ex- traction du minerai de fer sont visibles au nord. Dans la région de Kong où des fouilles réalisées font remonter l'apparition du fer au premier millénaire de notre ère, des traces de puits d'extraction sont également encore visibles ${ }^{15}$.

Les vestiges archéologiques de la métallurgie ancienne du fer laissent également des traces visibles utiles à l'étude et à la connaissance des techniques anciennes que sont les bas fourneaux de réduction et les scories.

L'étude archéologique de ces restes donne des informations intéressantes sur les types de fours, les différentes architectures, les modes et les matériaux de construction, les modes de fonctionnement, les types de souffleries ou de tuyères, les déchets et même les loupes.

Ceux-ci permettent également au chercheur de reconstituer les fourneaux en l'absence de données orales et écrites adéquates. Il peut ainsi recouper les données des traditions orales et des écrits avec celles des vestiges archéologiques afin de reconstituer les fourneaux si ceux-ci ne sont pas dans un bon état de conservation.

Des traces des fourneaux de réduction ont été signalées dans presque tous les pays d'Afrique subsaharienne. Des explorateurs, des administrateurs coloniaux, des amateurs, des scientifiques du début du $\mathrm{XX}^{\mathrm{e}}$ siècle ont fait le plus souvent les éloges de ces industries qui les fascinaient soit par les techniques utilisées, soit par les quantités de production. Ce qui a conduit souvent ces derniers à leur attribuer une origine étrangère. Nombre d'entre eux, devant l'évidence des faits, ont fini par accepter le haut degré de technicité, l'ancienneté et l'autochtonie de cette industrie en Afrique subsaharienne. Les recherches et écrits des scientifiques de ce siècle sont venus non seulement confirmer cette richesse métallurgique, mais aussi l'ancienneté et l'autochtonie de l'industrie des pays de l'Afrique subsaharienne.

La Côte d'Ivoire, malgré le fait qu'elle soit restée en marge du débat scientifique sur la métallurgie du fer, a mis en place une carte des centres métallurgiques du fer qui montre bien que tout le pays est parsemé de restes de scories et de fourneaux de réduction du minerai de 
fer $^{16}$. La zone forestière et la région savanicole ont connu cette industrie métallurgique.

$\mathrm{Au}$ niveau de la zone forestière, les centres les plus connus où l'on trouve des scories et des restes de fourneaux de réduction sont ceux d'Agboville, d'Oumé, d'Issia, Fresco et d'Assikoi ${ }^{17}$.

La zone savanicole, quant à elle, est connue pour sa richesse en matière de vestiges métallurgiques. Elle a comme centres sidérurgiques connus : Kong, Bouna, Korhogo, Bondiali, Odiénné, Touba, Mankono, Séguéla, Dabakala, Boudoukou ${ }^{18}$.

Au Burkina-Faso par contre, il a existé un véritable programme de recherche sur la métallurgie du fer. Ce qui a permis au pays de bénéficier d'une large couverture et d'avoir à disposition des résultats intéressants sur les données archéologiques de la métallurgie ancienne du fer ${ }^{19}$. Les vestiges de fourneaux au Burkina Faso sont souvent intacts et bien conservés à travers les âges. Les exemples les plus intéressants sont situés le plus souvent dans le nord du pays. Des fourneaux atteignent près de cinq mètres de hauteur avec à leur base de gros blocs de scories de différents aspects. On peut citer à titre d'exemple les fourneaux de Youba, de Kindbo au Yatenga, de Lantaga dans le Passoré etc. ${ }^{20}$. Les autres provinces ont aussi de véritables richesses en matière de vestiges archéologiques sur la métallurgie du fer. Ce qui a permis de mettre en place une carte des provinces et sites des vestiges métallurgiques du fer au Burkina-Faso.

Au Sénégal, les vestiges de la sidérurgie directe du fer sont aussi nombreux. Ils ont permis, à travers des études, de définir plusieurs étapes de l'histoire du fer. Les sites de l'âge du fer ont été identifiés et étudiés sur plusieurs localités, dont Sincu-Bara, Podor, Cuballel, Sylla, Ogo, Mbumba, Geedé, Namun, Ndalam etc. ${ }^{21}$.

En somme, les sites en Afrique subsaharienne sont très nombreux. C'est la preuve que l'on peut retrouver au sud du Sahara des éléments fondamentaux sur lesquels peuvent se fonder des études scientifiques pour aboutir à une histoire des techniques métallurgiques. Ces sources archéologiques peuvent être étudiées et recoupées avec les données des traditions orales, des do- cuments écrits anciens et récents, des analyses archéométriques pour comprendre, corriger, établir des typologies, des modes de fonctionnement, etc., sur les différents aspects de l'histoire des techniques métallurgiques du fer.

Les produits finis en fer sont aussi importants pour la compréhension de l'histoire des techniques métallurgiques. Ils peuvent être retrouvés chez les forgerons actuels et sur les sites d'habitat anciens. Des sites de métallurgie du fer sont souvent associés à des vestiges d'habitat et dans ce cadre précis, les fouilles de ces vestiges et l'étude des objets en fer trouvés en fouille permettent de faire une étude complète des différents stades de la chaîne opératoire de la sidérurgie directe du fer.

Tous ces vestiges archéologiques et historiques de l'activité métallurgique peuvent également donner des informations complémentaires essentielles que les méthodes classiques d'étude de la métallurgie sont incapables de nous fournir. Seule l'archéométrie peut répondre à certaines questions scientifiques fondamentales et constitue une des méthodes essentielles d'approche des vestiges métallurgiques qui sont souvent dépourvus d'informations de base.

L'archéométrie, en progrès constant, est une véritable chance pour les études des vestiges métallurgiques en Afrique. Du minerai jusqu'à l'élaboration des objets finis, il y a différentes étapes. Les métaux subissent des traitements thermiques et mécaniques. Au cours de ces étapes, les déchets et objets obtenus subissent des transformations physiques et chimiques qui laissent des traces au niveau de leurs microstructures. L'étude métallographique qui est l'approche structurale des objets métalliques permet d'appréhender les traitements thermomécaniques, les techniques de forge, le degré de technicité des forgerons, l'élaboration, l'utilisation et les réparations effectuées sur les objets. Ces modifications peuvent être étudiées dans le but de reconstituer cette chaîne opératoire. En l'absence d'informations sur les vestiges, cette étude fournit des résultats scientifiques fiables permettant, sur certains sites, de réduire le déficit d'informations. Il faut noter cependant que les résul- 
tats des examens « dépendent étroitement de la qualité de l'échantillonnage et des autres données fournies par les constats archéologiques sur le terrain ${ }^{22}$. C'est en ce sens que les travaux des spécialistes nationaux et étrangers représentent, ces dernières années, de véritables bases sur lesquelles les chercheurs peuvent s'appuyer non seulement pour les études sur la métallurgie de façon générale, mais aussi pour les approches les plus fines. Dans presque tous les pays de l'Afrique subsaharienne, des études scientifiques ont été faites avec des disparités d'un pays à l'autre selon les programmes et les équipes de recherche qui ont été mis sur pied. Les études archéométriques demandent une approche scientifique préalable des vestiges sur les sites archéologiques. C'est dans cet ordre d'idée que les problématiques traitées par les spécialistes sont très importantes pour les approches archéométriques. Des travaux scientifiques récents sur la métallurgie en Afrique subsaharienne ont déjà abordé les aspects archéométriques et surtout métallographiques. Ils ont donné des résultats intéressants sur le fonctionnement des fourneaux, les types de minerai, les différents aspects techniques, le niveau de développement technique des artisans, la reconstitution des chaînes opératoires, etc. ${ }^{23}$.

Néanmoins ces études archéométriques ont un inconvénient majeur, c'est le coût de leur réalisation qui constitue un véritable handicap pour les chercheurs africains, surtout que l'archéologie a du mal à réunir des fonds pour la recherche sur le terrain. Des accords au niveau institutionnel entre des universités africaines et des laboratoires spécialisés dans les études archéométriques seraient nécessaires pour la poursuite des recherches sur la métallurgie ancienne du fer. Avec le développement constant des techniques d'analyses archéométriques, les chercheurs se tournent de plus en plus vers celles-ci pour répondre à des problématiques plus fines. Aujourd'hui, bon nombre d'études sur la métallurgie ancienne du fer ne peuvent se faire sans l'apport de cette analyse incontournable, surtout pour les terrains africains où le plus souvent les vestiges archéologiques sont muets à cause du manque d'informations préalables importantes pour des approches préliminaires.
Les vestiges matériels sont des sources essentielles pour l'historien des techniques, spécialiste de la métallurgie du fer en afrique subsaharienne. Cependant les traditions orales, les sources immatérielles, renferment des informations historiques importantes qu'il faut savoir extraire des gestes et rites qui constituent un tout avec les volets techniques.

\section{Les sources orales}

L'intérêt de l'utilisation des traditions orales dans les études historiques et archéologiques pour les sociétés du sud du Sahara n'est plus à démontrer. Celles-ci constituent pour les chercheurs une source importante dont ils ne peuvent se défaire. Le problème essentiel est de mettre en place une base méthodologique afin de mener une bonne approche analytique des résultats des enquêtes orales. Leurs études comportent aussi bien des avantages que des inconvénients selon le traitement qu'elles subissent. Chaque société a ses particularités et les outils méthodologiques devraient tenir compte de ces différences au risque de passer à côté de faits scientifiques importants.

En Afrique subsaharienne, on a l'opportunité d'observer des techniques anciennes encore en usage. Il existe des personnes témoins qui peuvent les décrire afin que nous puissions les étudier. Les valeurs culturelles sont encore visibles et il n'y a pas eu de véritables ruptures culturelles jusqu'à nos jours dans nos sociétés traditionnelles.

$\mathrm{Au}$ niveau de l'histoire des techniques métallurgiques du fer, les résultats obtenus par l'utilisation des traditions orales sont inestimables.

De prime abord, c'est souvent par les traditions orales que les sites archéologiques qui ont trait à la métallurgie du fer sont découverts. Il s'agit des mines d'extraction du minerai de fer, des fourneaux de réduction et d'épuration du minerai et des sites d'habitat qui sont associés aux vestiges de la sidérurgie directe. De ce fait, elles constituent pour nous, historiens des techniques, des repères pour la découverte des sites qui sont les documents archéologiques sur lesquels le chercheur va asseoir son argumentation scientifique.

Pour ces sociétés subsahariennes, le recours aux tra- 
ditions orales ne sert pas seulement à la localisation des sites métallurgiques. Les informations données par les traditionnistes ${ }^{24}$ sont souvent, mis à part les vestiges archéologiques, les seuls éléments disponibles pour remonter les différentes étapes de la chaîne opératoire de la sidérurgie, de la recherche du minerai aux aspects socio-économiques de cette activité.

En effet, les documents écrits étant rares, il faut d'abord recueillir les données pour constituer cette documentation et les traditions orales peuvent nous renseigner sur certains aspects. Elles permettent le plus souvent de comprendre des sites archéologiques de la métallurgie ancienne du fer sur lesquels il n'existe aucune documentation écrite.

Les apports des traditionnistes à l'histoire des techniques sont divers. Lorsqu'ils sont eux-mêmes d'anciens métallurgistes et auteurs des vestiges métallurgiques, le chercheur dispose d'une source importante pour reconstituer la chaîne opératoire complète et connaître les techniques métallurgiques constituant le savoir-faire des forgerons et métallurgistes. Ainsi, les modes de prospection, d'exploitation du minerai, de fonctionnement des fourneaux et de traitement de la loupe, les types de fourneaux, les types de mines, les produits obtenus et les activités socio-économiques sont connus par les traditions orales et permettent de mieux appréhender les nombreux vestiges archéologiques encore visibles.

Lorsque les traditionnistes sont des métallurgistes n'ayant aucun lien avec les vestiges métallurgiques trouvés sur place, l'histoire du peuplement donne des indications qui peuvent aider à la datation relative des sites métallurgiques. Étant d'anciens spécialistes de la métallurgie du fer, ils peuvent apporter leur contribution à la compréhension des techniques et des données complexes sur les sites. En effet, l'Afrique subsaharienne a une diversité de techniques métallurgiques représentée par plusieurs formes de fourneaux de réduction qui demandent à être analysées et étudiées avec le concours des traditions anciennes.

Les données des traditionnistes, métallurgistes ou non, peuvent aussi être comparées aux vestiges archéologiques afin d'infirmer ou de confirmer certaines infor- mations, de suivre l'évolution des techniques métallurgiques dans le temps et dans l'espace par une étude comparative approfondie. En effet, les traditions orales décrivent des fourneaux ou des puits d'extraction, des techniques dont nous n'avons souvent aucun exemple en vue à cause de leur disparition. Une étude comparative peut nous conforter sur la valeur réelle de nos sources. C'est ainsi que les sources orales et archéologiques se rejoignent souvent pour lever certaines zones d'ombre. Un élément à souligner de ces traditions orales est l'importance des recueils et des observations des rituels et des gestes dans la production ancienne du fer, qui constituent parfois de véritables « véhicules d'histoire ».

\section{Les sources immatérielles}

Les études sur les aspects socio-culturels de la métallurgie ancienne du fer sont pour l'historien des techniques de véritables sources d'informations, surtout au niveau de la recherche des origines des forgerons et de la diffusion locale des techniques. Les méthodes d'approche dépendent des différentes problématiques de recherche et des périodes concernées.

En ce qui concerne les périodes les plus récentes, les aspects socio-cultuels qui constituent des sources immatérielles importantes peuvent être très utiles. Ceuxci sont de véritables « véhicules d'histoire » qu'il faut savoir déceler.

$\mathrm{Au}$ cours des libations, des rites renferment certaines informations historiques. Au Bulkiemdé (Burkina-Faso), lors des travaux de recherche sur l'histoire de la métallurgie ancienne du fer, ce sont des paroles prononcées lors des cérémonies rituelles avant la réduction du minerai de fer qui ont permis de remonter aux origines des forgerons, de leurs techniques, de découvrir les typologies de forges selon leur fonction, et les différentes voies de diffusions du métier de forgeron au plan local ${ }^{25}$.

En effet, les différentes étapes de la sidérurgie directe sont ponctuées par des rites, des gestes et des interdits qui, de prime à bord, paraissent comme de simples artifices pour le profane, mais en réalité, observés de près, on se rend compte que ce sont les fonda- 
tions même de cette industrie métallurgique ancienne. Comme l'écrivait Amadou Hampaté Ba : « En Afrique la vision de l'univers était une vision religieuse et globale et les actes de création y étaient rarement accomplis sans raison, sans préparation rituelle adéquate. Il n'y a pas le sacré d'un côté et le profane de l'autre, tout était lié... $~^{26}$. L'art du forgeron et du métallurgiste étant lié au mystère du feu, en Afrique, le sacré et la technique s'entremêlent. La production de la métallurgie ancienne du fer part de la recherche du minerai jusqu'à l'obtention du produit fini. À chaque étape interviennent des rites et des interdits ponctués de gestes souvent codifiés. Les exemples sont très nombreux, mais nous n'en exposons ici que quelques-uns.

Lors du chargement du fourneau pour la réduction du minerai de fer, le plus âgé des forgerons sur l'aire de réduction prononce des paroles rituelles et exécute des gestes après le sacrifice d'un animal (une poule) au fourneau. Il dit : " Je demande à la forge, aux maisons de forges l'autorisation de réduire mon minerai. Et si Dieu m'enlève de l'eau que j'ai le fer afin que les hommes aient des dabas, car si l'homme a à manger, il a la paix ». Les questions posées aux forgerons pour comprendre le sens de ces paroles prononcées lors des libations ont permis de découvrir des sources immatérielles importantes pour l'histoire de la métallurgie ancienne du fer dans le Bulkiemdé. Ainsi plusieurs types de forges ont été découverts avec des fonctions différentes les unes des autres et cela dans toutes les sociétés de forgerons dans la province du Bulkiemdé. Au total nous avons pu découvrir qu'il existait quatre types de forges. Ce sont : le kudkansanga, le kudkutgu, la forge sanctuaire et la forge protectrice.

« La forge » dont parle le forgeron lors des paroles rituelles à la réduction du minerai de fer désigne la grande forge du chef forgeron, appelée kudkansanga, constituée uniquement d'enclumes et d'objets rituels . On ne transforme pas la loupe en produits finis dans celle-ci. C'est la forge mère sous l'autorité du chef forgeron d'où sont tirés les pouvoirs mystiques et rituels des forges (kudkutgu) des autres forgerons qui transforment la loupe en produits finis dans leur forge res- pective. L'acceptation ou le refus de l'installation d'une forge par un forgeron et la radiation du métier de forgeron dans la région sont soumis à son avis. Elle relève de l'autorité du chef forgeron et coiffe toutes les autres forges que le traditionniste désigne sous le terme « maison de forge ». Celle-ci est en fait le kudkutgu (fig. 3 et 4), la forge où l'on transforme la loupe en produits finis et qui constitue par la même occasion un sanctuaire.

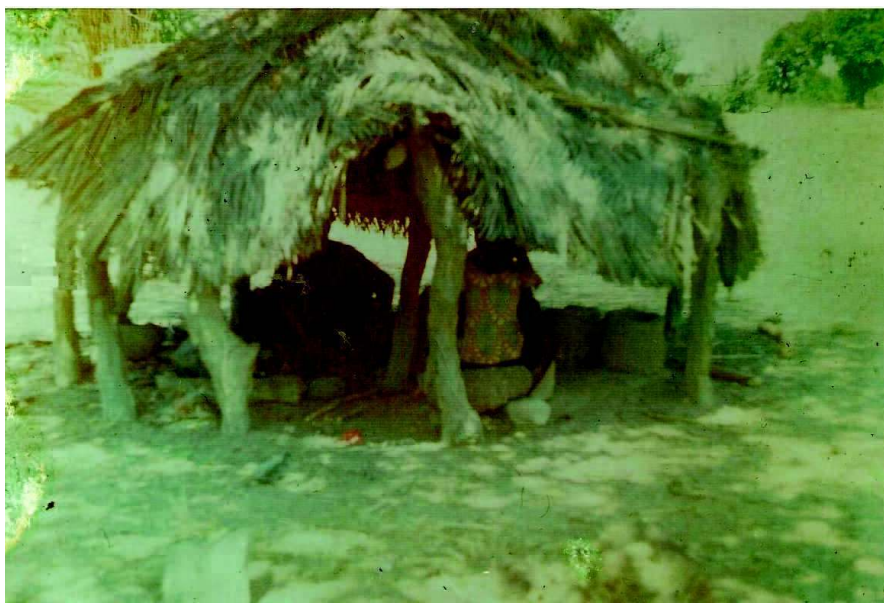

Fig. 3 : Vue d'ensemble du Kudkutgu

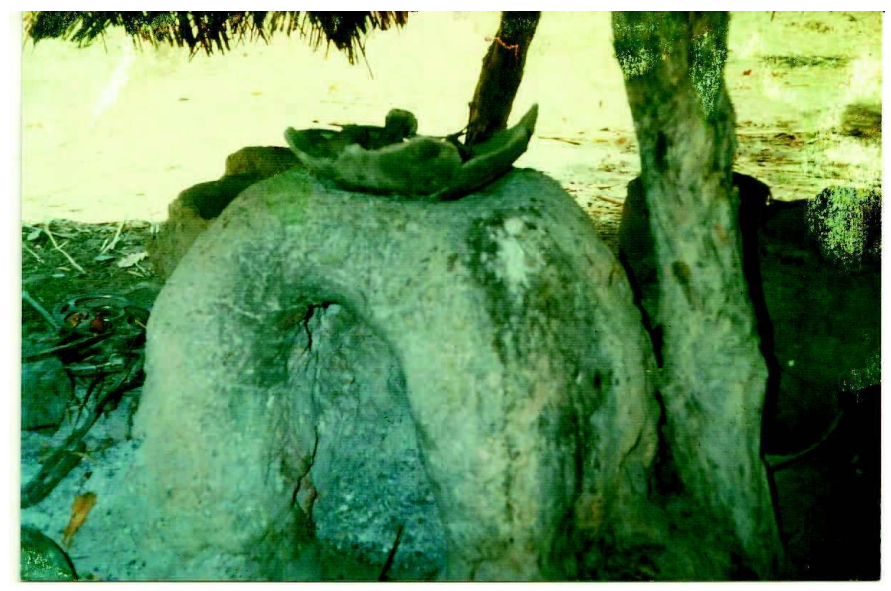

Fig. 4 : Foyer sanctuaire du Kudkutgu 
La forge sanctuaire quant à elle est en fait le kudkutgu appartenant à un forgeron qui a décidé d'arrêter le travail de transformation de la loupe à la forge. La tradition forgeronne interdisant de déterrer l'enclume qui est la pièce maitresse tant au niveau rituel que technique, le kudkutgu devient ainsi un sanctuaire où il doit continuer à faire des sacrifices aux dieux de la forge.

Le dernier type de forge, constitué uniquement d'une enclume, est un sanctuaire que des personnes non forgeronnes acquièrent (sous le conseil d'un devin) en échange d'un bœuf dans le but de protéger leur famille des esprits mauvais et des maladies. Ce sont des forges protectrices dont les propriétaires dépendent du chef forgeron (fig. 5). Ils peuvent y faire de petites réparations d'outils.

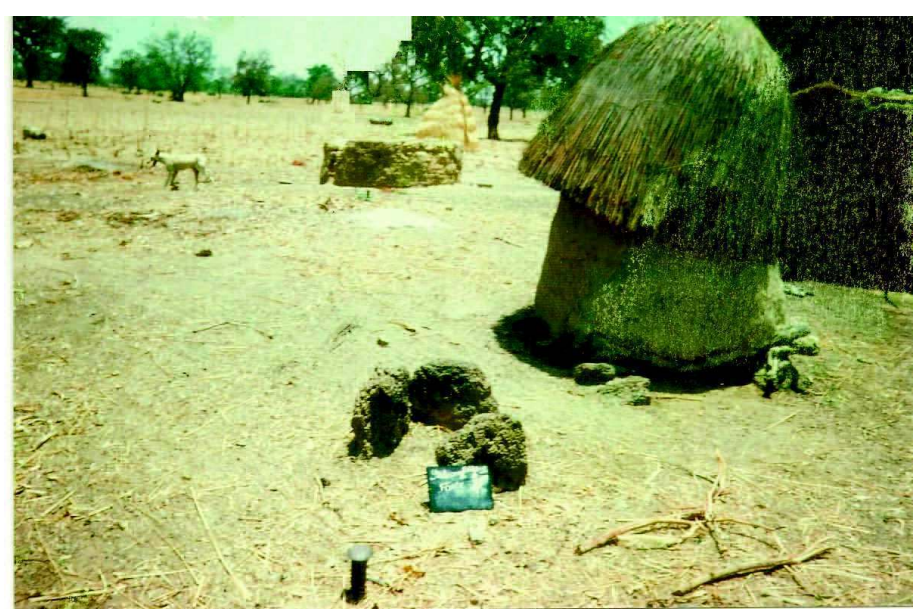

Fig. 5 : Forge protectrice constituée uniquement d'une enclume.

La typologie des forges a permis de comprendre également le mode de diffusion des techniques des forgerons et métallurgistes. Les forgerons peuvent être de différentes origines. On ne nait pas forgeron comme dans le Yatenga au nord du Burkina Faso où il existe un véritable système d'endogamie. Dans le Bulkiemdé, on devient forgeron par sa mère forgeronne ou simplement en apprenant les techniques et les rites liés au métier de la forge sans être forgeron de naissance.
Les interdits liés à la production du fer cachent également souvent des faits historiques importants. Au niveau des interdits sexuels, des éléments d'enquête montrent qu'il y a eu des catastrophes dans le passé, liées à des jalousies et à la convoitise des mêmes femmes, qui ont amené les métallurgistes à prendre des décisions ensuite érigées en interdits rituels et que beaucoup attribuent aux dieux de la forge. Les métallurgistes sont ainsi interdits de tous rapports sexuels avec les mêmes femmes. En effet, ceux-ci appartiennent à une communauté soucieuse de la bonne entente. Ils sont interdits de courtiser les mêmes femmes, qu'elles viennent de l'intérieur ou de l'extérieur du groupe, évitant ainsi les rivalités qui peuvent jouer sur la cohésion sociale du groupe. Tous ceux qui extraient le minerai et le réduisent au niveau du fourneau sont soumis aux mêmes règles sous peine de la colère des dieux qui entraine des éboulements de la mine et des échecs de la réduction.

Des raisons historiques de cet interdit existent. Dans la province du Sanguié à Réo, à l'ouest de celle du Bulkiemdé, plusieurs éboulements ont vu souvent des familles de forgerons disparaître. Les mines de Réo étaient réputées pour la bonne qualité de leur minerai et constituaient de grands centres d'extraction du minerai de fer pour les forgerons des villages du Bulkiemdé et des zones environnantes. Il fallait éviter les rivalités qui pouvaient entraîner des règlements de compte entre les mineurs et métallurgistes et cultiver ainsi l'esprit d'entraide et de solidarité.

Les sources immatérielles nous donnent des informations historiques importantes qui peuvent aider à reconstituer l'histoire du peuplement des forgerons et métallurgistes, les aspects techniques et socio-économiques de la métallurgie ancienne du fer. Cependant il faut les aborder avec une méthode rigoureuse qui permette de tirer des informations historiques de ces manifestations rituelles.

\section{Conclusion}

L'histoire de la métallurgie ancienne du fer en Afrique subsaharienne dépend essentiellement des 
sources et des méthodes appliquées. Il n'y a pas de civilisations plus enclines à écrire l'histoire que d'autres parce qu'elles ont connu l'écriture. La connaissance de l'écriture ne peut pas être le seul critère pour une bonne transmission de l'histoire en général et de l'histoire des techniques en particulier. Le choix des sources pour l'écriture de l'histoire des techniques dans chaque société est fondamental. En Afrique, les sociétés traditionnelles vénèrent la parole, qui est sacrée à tel point que les transmissions des savoirs qui se font de bouche à oreille sont surveillées et cadrées par des interdits et des sanctions divines selon les traditionnistes, même s'il existe quelques fois des déformations et des imperfections.

Dans les sociétés africaines subsahariennes également, les sources de l'écriture de l'histoire des techniques sont diverses et parfois spécifiques. Les gestes, les expressions des corps lors des cérémonies rituelles, les danses rituelles, etc. sont des sources immatérielles qui renferment des données historiques inestimables. L'absence d'écriture dans ces sociétés a développé d'autres formes de communication et de conservation des faits historiques et culturels qui peuvent être destinées aux profanes ou réservées aux seuls initiés.

Pour les sociétés qui ont connu très tôt l'écriture et qui bénéficient d'un grand nombre de documents écrits, les traditions orales et les sources immatérielles sont considérées comme des sources légères qui ne peuvent pas apporter des résultats scientifiques fiables, même pour les sociétés africaines au sud du Sahara dont les sources orales constituent quelquefois les seuls éléments disponibles pour l'approche historique. Cette étude nous montre qu'il est important de tenir compte d'un certain nombre de réalités spécifiques à chaque société, au risque de n'avoir qu'une partie des données et par conséquent de tronquer ou de fausser l'information historique recherchée. Pour les chercheurs spécialistes des sociétés africaines subsahariennes, les traditions orales, les sources immatérielles, les vestiges archéologiques, les documents écrits sont des sources importantes et complémentaires pour l'étude de l'histoire des techniques métallurgiques du fer en Afrique subsaharienne.
${ }^{1}$ COGHIAN H. H., « Some fresh aspects of the prehistoric metallurgy of copper », Antiquity, $\mathrm{n}^{\circ}$ 22, 1942, p 22-38; SHINNIE P. L. Meroe: a civilization of the Sudan, Londres, Thames and Hudson, coll. Ancient People and Places, $\mathrm{n}^{\circ}$ 25, 1967. TYLECOTE R. F., « The origin of iron smelting in Africa », West African Journal of Archaeology, vol $5, \mathrm{n}^{\circ} 1,1975$.

${ }^{2}$ BOCOUM H. (dir.), Aux origines de la métallurgie du fer en Afrique. Une ancienneté méconnue. Afrique de l'Ouest et Afrique centrale, UNESCO, 2002, $240 \mathrm{p}$.

${ }^{3}$ BOCOUM H., La métallurgie du fer au Sénégal : approche archéologique, technologique et historique, thèse de doctorat, Université Paris 1 Panthéon-Sorbonne, 1986, 333p. ; BOCOUM H., L'âge du fer au Sénégal, Dakar, Institut fondamental d'Afrique noire, Université Cheikh-Anta-Diop, 2000 ; COULIBALY E., Savoir et savoirfaire des anciens métallurgistes d'Afrique occidentale : procédés et techniques de la sidérurgie directe du fer dans le Bwamu (Burkina Faso-Mali), Karthala, 2006, 422p. ; KIÉNON-KABORE T. H. La métallurgie ancienne du fer au Burkina Faso: Province du Bulkiemdé. Approche ethnologique, historique, archéologique et métallographique. Un apport à l'histoire des techniques métallurgiques en Afrique, l'Harmattan, 2003, 328p. ; KIETHEGA J. B., La métallurgie lourde du fer au Burkina-Faso. Une technologie à l'époque précoloniale, Karthala, 2009, 500p.

${ }^{4}$ BINGER L. G., Du Niger au golfe de Guinée par le pays de Kong et le Mossi. Tome I, Paris, Hachette 1892, 513p.

${ }^{5}$ MENIAUD J., Haut Sénégal Niger. Soudan Français, Paris, Émile Larose, 1912.

${ }^{6}$ YANDIA F., « La métallurgie du fer en République Centrafricaine d'après les sources écrites. », dans BENOIT P. et FLUZIN P. (dir.), Paléométallurgie du fer et cultures. Symposium International du Comité pour la Sidérurgie ancienne, Vulcain, 1995, pp. 151-154.

7 DOGNON H., «L'Histoire du fer au Bénin », dans BENOIT P. et FLUZIN P. (dir.), 1995, op. cit., pp. 155-157.

${ }^{8}$ FOFANA L., « Problématique de la métallurgie ancienne du fer en Côte d'Ivoire précoloniale. », West African Journal of Archaeology (WAJA), imprints of West Africa's Past, 1993, pp. 257-266.

9 BOCOUM H., «L'Archéologie française en Afrique de l'ouest : rétrospectives et enjeux. », dans BAZZANA A. et BOCOUM H. (dir.), Du nord au sud du Sahara cinquante ans d'archéologie française en Afrique de l'ouest et au Maghreb. Bilan et perspectives, SEPIA 2004, pp. 29-36.

${ }^{10}$ BOCOUM H., 2002, op.cit., p. 21.

${ }^{11}$ QUECHON G., "Les datations de la métallurgie du fer à Termit (Niger) : leur fiabilité, leur signification », dans BOCOUM H., 2002, op. cit., pp. 105-114.

${ }^{12}$ KIETHEGA J. B., 2009, op. cit., p. 145 .

${ }^{13}$ KIÉNON-KABORE T. H, 2003, op.cit., pp. 76 et 77 .

${ }^{14}$ KIETHEGA J. B., 1996, op. cit., p. 50.

${ }^{15}$ FOFANA L., 1993, op. cit., p. 257.

${ }^{16}$ Ibidem, p. 258.

${ }^{17}$ KABORE-KIÉNON T. H., « Problématique de la métallurgie an- 
cienne du fer sur la sphère Akan de Côte d'Ivoire. Une contribution à l'Histoire des techniques métallurgiques. ", Godo Godo, n 16 , 2006.

${ }^{18}$ FOFANA L., 1993, op. cit.

${ }^{19}$ Le Laboratoire d'archéologie de l'Université de Ouagadougou, à travers le projet « histoire du fer au Burkina Faso », a pu effectivement couvrir une grande partie du pays. Plusieurs mémoires de maîtrise ont été soutenus, ainsi que trois thèses de doctorat, dont une thèse d'État. Ce sont, pour les thèses de doctorat : KIETHEGA J. B., 1996, op. cit., COULIBALY E., 2006, op. cit., et KIÉNON-KABORE T. H., op. cit., 2003.

${ }^{20}$ KIETHEGA J. B., op cit, p. 376.

${ }^{21}$ BOCOUM H., "La métallurgie du fer au Sénégal des origines au $\mathrm{XX}^{\mathrm{e}}$ siècle. Esquisse d'une évolution des techniques de réduction. ", dans BENOIT P. et FLUZIN P. (dir.), op. cit., 1995, pp. 143-149.

${ }^{22}$ FLUZIN Ph., " La chaîne opératoire en sidérurgie : matériaux archéologiques et procédés. Apport des études métallographiques », dans BOCOUM H. (dir.), 2002, op.cit., pp. 59-91.

${ }^{23}$ BOCOUM H ., 1986, op. cit. ; COULIBALY E., 2006, op.cit. ; YANDIA F., La métallurgie du fer en République Centrafricaine du $X V I I^{e}$ au XX $X^{e}$ siècle, thèse de doctorat, Université Paris 1 PanthéonSorbonne, 1994. KIÉNON-KABORE T. H., 2003, op. cit.

${ }^{24}$ Les traditionnistes sont les détenteurs des traditions ancestrales. Ils peuvent traiter de différents sujets. En sidérurgie directe, les traditionnistes peuvent être des forgerons, des métallurgistes ou toutes autres personnes pouvant donner des informations sur cette industrie.

${ }^{25}$ KIÉNON T. H., L'exploitation traditionnelle du fer à Ralo (Province du Bulkiemdé, Burkina Faso), mémoire de maitrise, Université de Ouagadougou, 1990, 163p, p. 90.

${ }^{26}$ BA A. H., " En Afrique, cet art où la main écoute », Le courrier de l'UNESCO, février 1976.

\section{Bibliographie}

BA A. H., « En Afrique, cet art où la main écoute », Le courrier de l'UNESCO, février 1976.

BOCOUM H., La métallurgie du fer au Sénégal : approche archéologique, technologique et historique, thèse de doctorat, Université Paris 1 Panthéon-Sorbonne, 1986, 333p.

BOCOUM H., " La métallurgie du fer au Sénégal des origines au $\mathrm{XX}^{\mathrm{e}}$ siècle. Esquisse d'une évolution des techniques de réduction ", dans BENOIT P. et FLUZIN P. (dir.), Paléométallurgie et cultures. Symposium International du Comité pour la Sidérurgie Ancienne, éd. Vulcain, 1995, pp. 143-149.

BOCOUM H. (dir.), Aux origines de la métallurgie du fer en Afrique. Une ancienneté méconnue, éd. UNESCO, 2002, $240 \mathrm{p}$.

BOCOUM H., « L'Archéologie Française en Afrique de l'Ouest : rétrospectives et enjeux » dans BAZZANA A. et BOCOUM H. (dir.),
Du nord au sud du Sahara cinquante ans d'archéologie Française en Afrique de l'Ouest et au Maghreb. Bilan et perspective, éd. SEPIA, Paris, 2004, p. 29-36.

CAPITAINE BINGER, Du Niger au golfe de Guinée par le pays de Kong et le Mossi, Paris, éd. Hachette, 1892.

COGHIAN H.H., " Some fresh aspects of the prehistoric metallurgy of copper », Antiquity, n' ${ }^{\circ} 2$, 1942, pp. 22-38.

COULIBALY E., Savoir et savoir-faire des anciens métallurgistes d'Afrique occidentale : procédés et techniques de la sidérurgie directe du fer dans le Bwamu (Burkina Faso-Mali) éd. Karthala, 2006, 422p.

DOGNON H., « L'Histoire du fer au Bénin », dans BENOIT P. et FLUZIN P. (dir.), Paléométallurgie et cultures. Symposium International du Comité pour la Sidérurgie Ancienne, éd. Vulcain, 1995, pp. 155-157.

FLUZIN Ph., « La chaîne opératoire en sidérurgie : matériaux archéologiques et procédés. Apport des études métallographiques », dans BOCOUM H. (dir.), Aux origines de la métallurgie du fer en Afrique. Une ancienneté méconnue, éd. UNESCO, 2002, 240p, pp. 59-91.

FOFANA L., " Problématique de la métallurgie ancienne du fer en Côte d'Ivoire précoloniale », West African Journal of Archaeology (WAJA), imprints of West Africa's Past, 1993, p 257-266.

KIÉNON T. H., L'exploitation traditionnelle du fer à Ralo (Province du Bulkiemdé Burkina Faso), mémoire de maitrise, Université de Ouagadougou, 1990, 163p.

KIÉNON-KABORE T. H., La métallurgie ancienne du fer au Burkina Faso: Province du Bulkiemdé. Approche ethnologique, historique, archéologique et métallographique. Un apport à l'histoire des techniques métallurgiques en Afrique, éd. de l'Harmattan, 2003, 328p.

KABORE-KIÉNON T. H., « Problématique de la métallurgie ancienne du fer sur la sphère Akan de Côte D'Ivoire. Une contribution à l'histoire des techniques métallurgiques », Godo Godo, n¹6, 2006.

KIETHEGA, J. B., La métallurgie lourdes du fer au Burkina Faso, tomes 1 et 2, thèse de doctorat d'État, Université Paris 1 PanthéonSorbonne, 1996.

KIETHEGA J. B., La métallurgie lourde du fer au Burkina-Faso. Une technologie à l'époque précoloniale, éd. Karthala, 2009, 500 p.

MARTINELLI B., "Fonderie ouest Africaines. Classement comparatif et tendances ", MSH, Revue Techniques et cultures, $1993, \mathrm{n}^{\circ} 21$.

MARTINELLI B., " Au seuil de la métallurgie intensive. Le choix de la combustion lente dans la boucle du Niger (Burkina-Faso et Mali) », dans BOCOUM H. (dir.), Aux origines de la métallurgie du fer en Afrique, éd. UNESCO, 2002.

MENIAUD J., Haut Sénégal Niger. Soudan Français, Paris, Émile Larose, 1912.

QUECHON G., « Les datations de la métallurgie du fer à Termit (Niger) : leur fiabilité, leur signification », dans BOCOUM H. (dir.), Aux origines de la métallurgie du fer en Afrique, éd. UNESCO, 2002, pp. 105-114.

SHINNIE P. L., « Meroe: a civilization of the Sudan », Ancient People and Places, 25, Londres, Thames and Hudson, 1967. 
TYLECOTE, R. F., « The origin of iron smelting in Africa », West African Journal of Archaeology, vol 5, ${ }^{\circ}{ }^{1}$, 1975, pp. 1-9.

YANDIA F., " La métallurgie du fer en République Centrafricaine d'après les sources écrites », dans BENOIT P. et FLUZIN P. (dir.), Paléométallurgie du fer et cultures, Symposium International du Comité pour la Sidérurgie ancienne, éd. Vulcain 1995, pp. 151-154. YANDIA F., La métallurgie traditionnelle du fer en Afrique Centrale, éd. de l'Harmattan, 2001, 320 p. 